\title{
NONLINEAR DIAGNOSTICS USING AC DIPOLES *
}

\author{
S. Peggs, BNL, Upton, NY
}

There are three goals in the accurate nonlinear diagnosis of a storage ring. First, the beam must be moved to amplitudes many times the natural beam size. Second, strong and long lasting signals must be generated. Third, the measurement technique should be non-destructive.

Conventionally, a single turn kick moves the beam to large amplitudes, and turn-by-turn data are recorded from multiple beam position monitors (BPMs) [1-6]. Unfortunately, tune spread across the beam causes the center of charge beam signal to "decohere" on a time scale often less than 100 turns. Filamentation also permanently destroys the beam emittance (in a hadron ring). Thus, the "strong single turn kick" technique successfully achieves only one out of the three goals. AC dipole techniques can achieve all threc. Adiabatically excited AC dipoles slowly move the beam out to large amplitudes. The coherent signals then recorded last arbitrarily long. The beam maintains its original emittance if the AC dipoles are also turned off adiabatically, ready for further use.

The AGS already uses an $R F$ dipole to accelerate polarized proton beams through depolarizing resonances with minimal polarization loss [7]. Similar AC dipoles will be installed in the horizontal and vertical planes of both rings in RHIC [8]. The RHIC AC dipoles will also be used as spin flippers, and to measure linear optical functions [9].

\section{LINEAR MOTION}

Horizontal motion is described using complex phasors

$$
z \equiv x^{\prime}+i x=a e^{i \phi}
$$

so that the unperturbed one turn motion is just

$$
z_{t+1}=R z_{t}
$$

where $R=\exp \left(i 2 \pi Q_{X}\right)$. Here $Q_{X}$ is the betatron tune, and the normalized coordinates $x$ and $x^{\prime}$ both have the dimensions of length. An AC dipole just after the reference point gives a real normalized angular kick on turn $t$ of

$$
\Delta z_{t}=\Delta x^{\prime}=\delta \cos \left(2 \pi Q_{D} t+\psi_{0}\right)
$$

where $Q_{D}$ is the drive tune and $\psi_{0}$ is the initial phase. The AC dipole strength is $\delta=(B L /(B \rho)) \beta_{D}$, where $B L$ is the integrated field amplitude, $(B \rho)$ is the rigidity, and $\beta_{D}$ is the Twiss function at the dipole.

If $z=z_{0}$ just before the first dipole kick, then the net displacement phasor on turn $T$ is

$$
z_{T}=R^{T} z_{0}+\left(R^{T} \Delta z_{0}+R^{T-1} \Delta z_{1} \cdots+R^{1} \Delta z_{T-1}\right)
$$
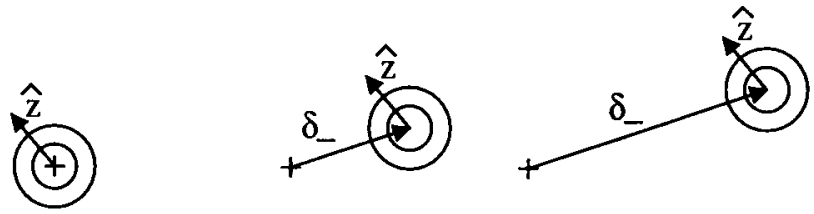

Figure 1: Adiabatic excitation of an AC dipole, in the rotating frame. The circles represent single particle motion.

The exact general solution for linear motion is [10]

$$
z_{T}=\widehat{z} e^{i 2 \pi Q_{X} T}+\delta_{-} e^{i 2 \pi Q_{D} T}-\delta_{+} e^{-i 2 \pi Q_{D} T}
$$

where $\widehat{z}=z_{0}-\delta_{-}+\delta_{+}$is a constant given by the initial conditions, and the complex $\mathrm{AC}$ dipole strengths are

$$
\begin{aligned}
& \delta_{-}=\frac{\delta}{4} \frac{\exp \left(-i\left[\pi Q_{-}-\psi_{0}\right]\right)}{\sin \left(\pi Q_{-}\right)} \\
& \delta_{+}=\frac{\delta}{4} \frac{\exp \left(i\left[\pi Q_{+}-\psi_{0}\right]\right)}{\sin \left(\pi Q_{+}\right)}
\end{aligned}
$$

where $Q_{-}=Q_{D}-Q_{X}$ and $Q_{+}=Q_{D}+Q_{X}$.

The oscillating closed orbit is defined as that orbit which exactly repeats itself after one modulation period. The solution on turn $T$ is obtained by putting $\hat{z}=0$, so that

$$
z_{C O}=\delta_{-} e^{i 2 \pi Q_{D} T}-\delta_{+} e^{-i 2 \pi Q_{D} T}
$$

generally following a tilted ellipse, not a circle, in normalized phase space. The semi-minor and semi-major axes are ||$\delta_{-}|-| \delta_{+}||$and $\left|\delta_{-}\right|+\left|\delta_{+}\right|$long. In practice the aspect ratio of the ellipse is close to 1 when the AC dipole is driven at a tune close to the fractional betatron tune $\left(Q_{-} \approx 0\right)$. When $\left|\delta_{-}\right| \gg\left|\delta_{+}\right|$the approximate motion is

$$
z_{T} \approx \widehat{z} e^{i 2 \pi Q_{X} T}+\delta_{-} e^{i 2 \pi Q_{D} T}
$$

Motion in the rotating frame, which rotates with the $\mathrm{AC}$ dipole drive at $2 \pi Q_{D}$ per turn, is denoted by over-bars. Assuming the previous approximation to be accurate,

$$
\bar{z}_{T}=\delta_{-}+\widehat{z} e^{-i 2 \pi Q_{-} T}
$$

That is, a test particle slowly circulates the vector $\delta_{-}$at a radius of constant length $|\widehat{z}|$, as illustrated in Fig. 1 .

When a bunch is considered, a distribution of $\widehat{z}$ values must be used. A smoothly distributed beam has $\langle\widehat{z}\rangle=0$ and $\left\langle\widehat{z}^{2}\right\rangle=2 \beta \epsilon_{u}$ where \langle\rangle represents a bunch average, and $\epsilon_{u}$ is the unnormalized root mean square emittance. The center of charge motion in the non-rotating frame is just

$$
\left\langle z_{T}\right\rangle=\delta_{-} e^{i 2 \pi Q_{D} T}
$$


Thus, the coherent bunch response to an adiabatically driven AC dipole has a constant amplitude

$$
a_{C O H}=\left|\delta_{-}\right|=\frac{1}{4}\left|\frac{\delta}{\sin \left(\pi Q_{-}\right)}\right|
$$

This has been quantitatively confirmed in the AGS [7].

Any bunch tune spread (due to non-zero chromaticity or nonlinear detuning) trivially modifies the rate of advance around the $\delta_{-}$vector for different particles. Less trivially, the tune spread also modifies $\delta_{-}$, which is a function of $Q_{-}$(see Eqn. 6). This is not a practical problem if $Q_{D}$ is sufficiently far outside the bunch tune spectrum.

\section{SHEAR MOTION - DETUNING}

The total approximate one turn difference map is

$$
\begin{aligned}
\Delta J= & \frac{\delta}{\sqrt{\beta_{D}}} \sqrt{2 J} \cos \left(2 \pi Q_{D} t\right) \cos (\phi) \\
\Delta \phi=- & \frac{\delta}{\sqrt{\beta_{D}}} \frac{1}{\sqrt{2 J}} \cos \left(2 \pi Q_{D} t\right) \sin (\phi) \\
& +2 \pi\left(Q_{X 0}+\alpha J\right)
\end{aligned}
$$

where $\psi_{0}$ is set to 0 , and the action $J=a^{2} / 2 \beta_{D}$ has the dimensions of length. Detuning with action is present, proportional to $\alpha J$, since (if $\delta=0$ )

$$
Q_{X}(J) \equiv\langle\Delta \phi\rangle / 2 \pi=Q_{X 0}+\alpha J
$$

where \langle\rangle represents a time average. A one turn discrete Hamiltonian $H_{1}$ concisely describes this motion, through

$$
\left(\begin{array}{c}
\Delta J \\
\Delta \phi
\end{array}\right)=\left(\begin{array}{r}
-\dot{\partial} H_{1} / \partial \phi \\
\partial H_{1} / \partial J
\end{array}\right)
$$

Since $H_{1}$ represents a difference map, and not continuous differential motion, it is not (necessarily) even approximately a constant of the motion. In the case at hand

$$
H_{1}=2 \pi\left(Q_{X 0} J+\frac{\alpha}{2} J^{2}\right)-\frac{\sqrt{2} \delta}{\sqrt{\beta_{D}}} J^{1 / 2} \cos \left(2 \pi Q_{D} t\right) \sin (\phi)
$$

This Hamiltonian is marred by its time dependence.

A canonical transformation to the rotating frame is achieved by applying the generating function

$$
W(\bar{J}, \phi, t)=\bar{J} \phi-2 \pi Q_{D} t \bar{J}
$$

New and old action-angle coordinates are related through

$$
\begin{aligned}
& J \equiv \partial W / \partial \phi=\bar{J} \\
& \bar{\phi} \equiv \partial W / \partial \bar{J}=\phi-2 \pi Q_{D} t
\end{aligned}
$$

If one turn motion is small $\left(Q_{-} \approx 0\right)$, the transformation $\bar{H}_{1} \equiv H_{1}+\partial W / \partial t$ (averaged over many turns) yields

$$
\bar{H}_{1}=2 \pi\left(\frac{\alpha}{2} \bar{J}^{2}-Q_{-} \bar{J}\right)-\frac{\delta}{\sqrt{2 \beta_{D}}} \bar{J}^{1 / 2} \sin (\bar{\phi})
$$

$\bar{H}_{1}$ is independent of time, and is a good approximation to a constant of the motion. The fixed points are given by

$$
\begin{aligned}
\bar{\phi}_{F P} & = \pm \frac{\pi}{2} \\
0 & =2 \pi\left(\alpha \bar{J}_{F P}-Q_{-}\right) \mp \frac{\delta}{2 \sqrt{2 \beta_{D}}} \frac{1}{\bar{J}_{F P}^{1 / 2}}
\end{aligned}
$$

In general there is either one stable fixed point, or one unstable and two stable fixed points [7]. When detuning is absent $(\alpha=0)$ the fixed point amplitude is

$$
a_{F P}=\frac{1}{4 \pi}\left|\frac{\delta}{Q_{-}}\right|
$$

agreeing with Eqn. 11 when $Q_{-}$is small!

\section{NONLINEAR MOTION IN 2-D}

The action-angle time series $\left(J_{x}, \phi_{x}, J_{z}, \phi_{z}\right)_{t}$ is derived from turn-by-turn data $\left(x_{1}, x_{2}, y_{1}, y_{2}\right)_{t}$ recorded at 2 horizontal and 2 vertical BPMs. This requires the empirical adjustment of the $\beta$ function ratio of each BPM pair, of the phase advance of each pair, and of the closed orbit error at every BPM, in a process which also corrects for the elliptical motion of the oscillating closed orbit $[1,3,6]$.

The general 2-D one turn discrete Hamiltonian is

$$
\begin{aligned}
H_{1} & =2 \pi Q_{X 0} J_{x}+2 \pi Q_{Y 0} J_{y} \\
& +\sum_{i j k l} V_{i j k l} J_{x}^{i / 2} J_{y}^{j / 2} \sin \left(k \phi_{x}+l \phi_{y}+\phi_{i j k l}\right)
\end{aligned}
$$

where the appropriate set of indices $(i j k l)$ depends on the dominant nonlinearities [2]. Only in the simplest of models can $V_{i j k l}$ and $\phi_{i j k l}$ be predicted analytically. The horizontal action time series is then

$$
J_{x}(t)=J_{x 0}-\sum_{i j k l} \frac{k V_{i j k l} J_{x 0}^{i / 2} J_{y 0}^{j / 2}}{2 \sin \left[\pi Q_{k l}\right]} \sin \left(2 \pi Q_{k l} t+\phi_{0 i j k l}\right)
$$

A single harmonic dominates if $Q_{k l}=k Q_{X}+l Q_{Y}$ approaches an integer for some $(k, l)$ pair. With coherent bunch motion, $Q_{X}$ and $Q_{Y}$ represent the drive tunes of simultaneous horizontal and vertical AC dipoles, and $J_{x 0}$ and $J_{y 0}$ represent the average (fixed point) actions.

The Discrete Fourier Transform (DFT) of a long action time series generates action harmonic coefficients

$$
D_{x k l}=\frac{-k}{2 \sin \left[\pi Q_{k l}\right]} \sum_{i j} V_{i j k l} J_{x 0}^{i / 2} J_{y 0}^{j / 2} e^{i \phi_{0} j k l}
$$

The value of a coefficient ( $D_{x k l}$ or $D_{y k l}$ ) depends on the $J_{x 0}$ and $J_{y 0}$ values used in that measurement. Multiple measurements on a grid of $\left(J_{x 0}, J_{y 0}\right)$ values can be used to recover a complete set of $V_{i j k l}$ and $\phi_{0 i j k l}$ values.

Sometimes the motion is summarized by smear statistics [2]. The horizontal smear $s_{x x}$ is given by

$$
s_{x x}^{2} \equiv \frac{\left\langle a_{x} a_{x}\right\rangle}{\left\langle a_{x}\right\rangle\left\langle a_{x}\right\rangle}-1=\sum_{i j k l} \frac{k^{2} V_{i j k l}^{2} a_{x 0}^{2 i-4} a_{y 0}^{2 j}}{2^{i+j+3} \sin ^{2}\left[\pi Q_{k l}\right]}
$$



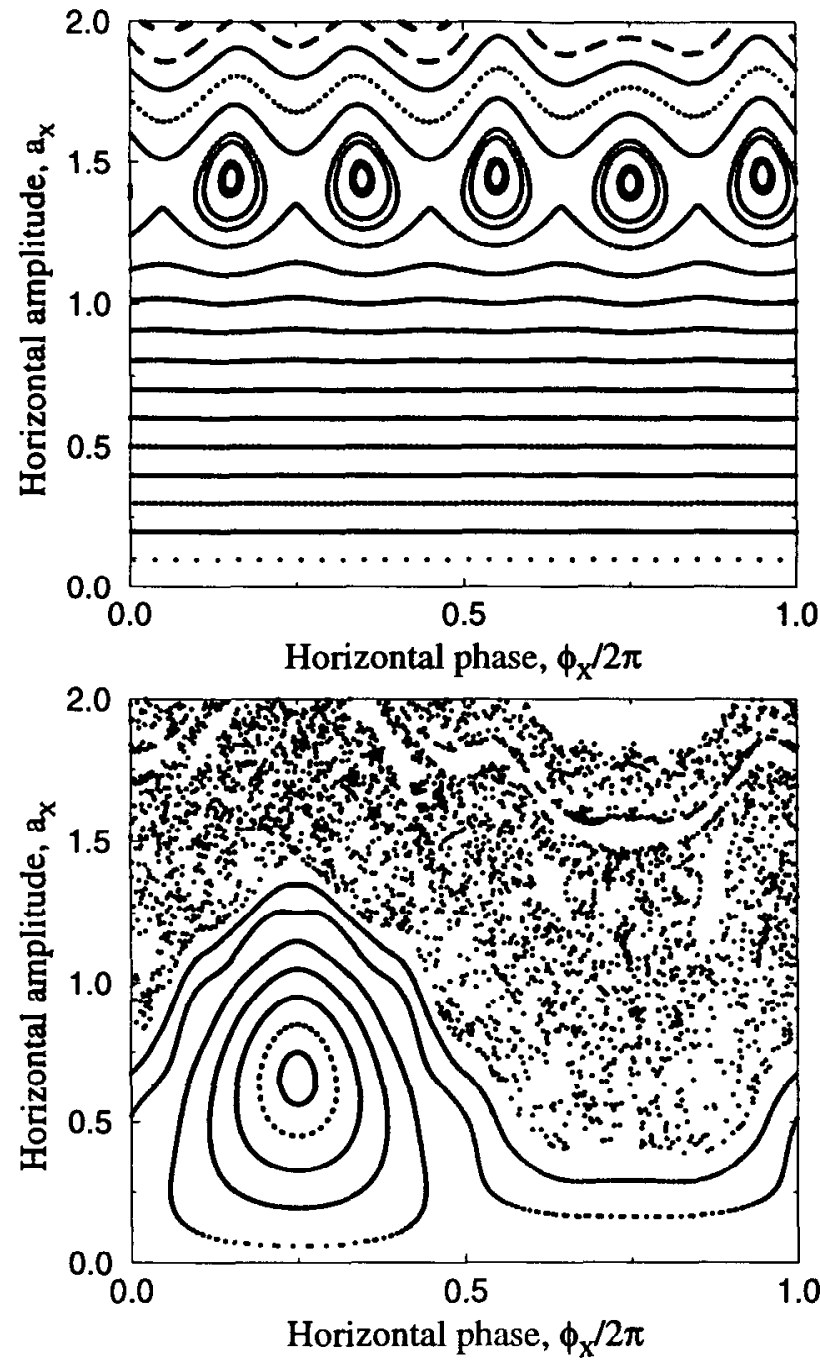

Figure 2: 1-D motion near a decapole driven resonance with an AC dipole OFF (TOP) or ON (BOTTOM).

(assuming $\beta_{x}=\beta_{y}=1$ ). Similar expressions exist for the vertical and correlation smears, $s_{y y}$ and $s_{x y}$. Predicted and measured smears agree well in controlled experiments with a small number of dominant nonlinearities $[3,4]$.

\section{A NUMERICAL EXPERIMENT}

Figure 2 illustrates a simple 1-D numerical experiment. Detuning (from $Q_{x}=0.591$ to 0.609 between $a_{x}=0$ and 2.0) is driven by three octupoles, arranged to minimize octupole driven resonances [6]. A single decapole drives the $Q_{x}=3 / 5$ resonance, generating a chain of 5 islands at $a_{R E S} \approx 1.4$ in the TOP figure. The AC dipole tune $Q_{D}=59 / 101 \approx 0.584$ allows a plotting period of 101 turns to be used in the BOTTOM figure. A coherent $A C$ dipole $O N$ signal is simulated by launching a single particle at the fixed point in the BOTTOM figure, to generate a turn-by-turn "BPM" time series. The goal is to show that this time series closely resembles that obtained with the same launch coordinates, but with the AC dipole OFF.

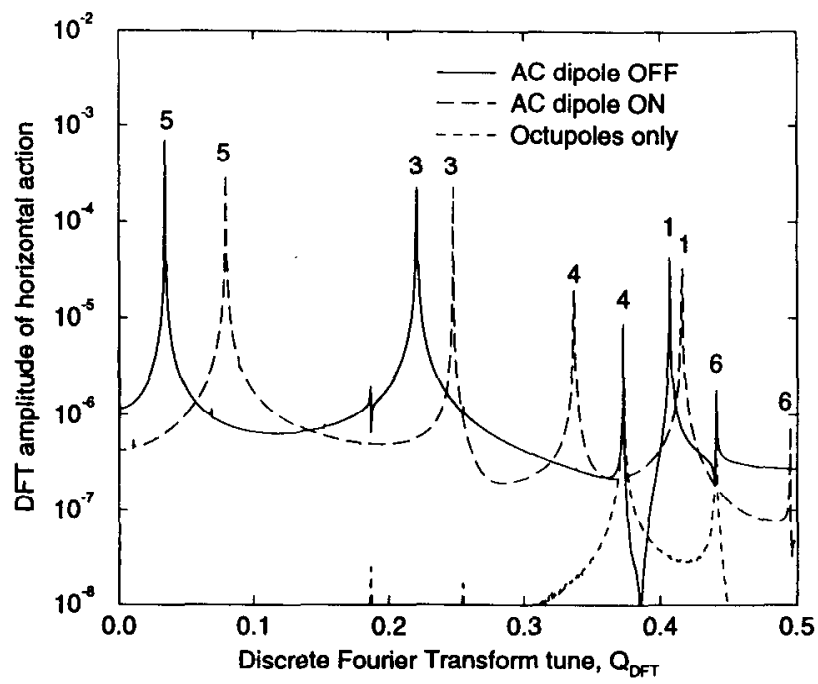

Figure 3: Discrete Fourier Transforms of the horizontal action of a test particle in the numerical experiment.

Figure 3 shows action DFTs with the AC dipole OFF and $\mathrm{ON}$. The harmonic peaks shift because $Q_{X} \approx$ $0.593(0.584)$ in the OFF (ON) case. Nonetheless, the $D_{x 50}$ and $D_{x 30}$ values derived from both data sets are closely consistent, after correcting for the $1 / \sin \left[\pi Q_{k 0}\right]$ dependence in Eqn. 24. This implies that single particle Hamiltonian values $V_{i j k l}$ and $\phi_{0 i j k l}$ can indeed be derived from measurements of coherent motion driven by $\mathrm{AC}$ dipoles.

\section{ACKNOWLEDGMENTS}

Many thanks to Mei Bai, Rhianna Bianco, Wolfram Fischer, Todd Satogata, and Chunmei Tang.

\section{REFERENCES}

[1] A. Chao et al, PRL 61 (1987) 2752; T. Chen et al, PRL 68 (1992) 33; T. Satogata et al, PRL 68 (1992) 1838

[2] S. Peggs, Proc. 2nd ICFA workshop, CERN 88-04, and SSC175 (1988)

[3] N. Merminga, Ph.D. Thesis, U. Michigan (1989)

[4] M.Y. Li, Ph.D. Thesis, U. Houston (1990)

[5] S.Y. Lee et al, PRL 67 (1991) 3768; D.D. Caussyn et al, PRA 46 (1992) 7942; W. Fischer, Dissertation, U. Hamburg (1995); W. Fischer et al, PRE 55 (1997) 3507

[6] T. Satogata, Ph.D. Thesis, Northwestern U. (1993)

[7] M. Bai et al, PRE 56 (1997) 6002; M. Bai, Ph.D. Thesis, U. Indiana (1999); M. Bai et al, Beam manipulation with an $\mathrm{RF}$ dipole, these proceedings (1999)

[8] B. Parker et al, Design of an AC dipole for use in RHIC, these proceedings (1999)

[9] P. Castro-Garcia, Doctoral Thesis, U. de Valencia (1996)

[10] S. Peggs and C. Tang, RHIC/AP/159, Upton, (1998) 